\title{
Satellite delay spells shortage of star data
}

\section{Alison Abbott, Munich}

The recent decision to delay the launch of a German satellite has left astrometry - the study of the exact position of objects in space - facing a prolonged data drought.

The satellite, known as DIVA, was scheduled to take off in 2004 on a mission to catalogue the positions of stars with unprecedented accuracy. But its launch has now been put back to at least 2007 .

Astrometry specialists say that this delay - which closely follows the cancellation of NASA's FAME, a similar mission also scheduled to launch in 2004 (see Nature 415, 249; 2002) - will deprive them of useful data for nearly a decade. The next big astrometry mission, GAIA, which is being run by the European Space Agency (ESA), is expected to launch sometime between 2010 and 2014 .

The astrometry community has been getting by on data collected between 1989 and 1993 by ESA's Hipparcos mission. DIVA and FAME were supposed to breathe new life into the community, attracting and training young researchers in preparation for the masses of data that GAIA will eventually deliver.

Both DIVA and FAME were designed to map tens of millions of stars, compared with the 100,000 contained in the Hipparcos catalogue. But GAIA should position as many as a billion stars, and is also expected to locate about 40,000 large planets outside the Solar System.

The catalogues prepared by such mis-

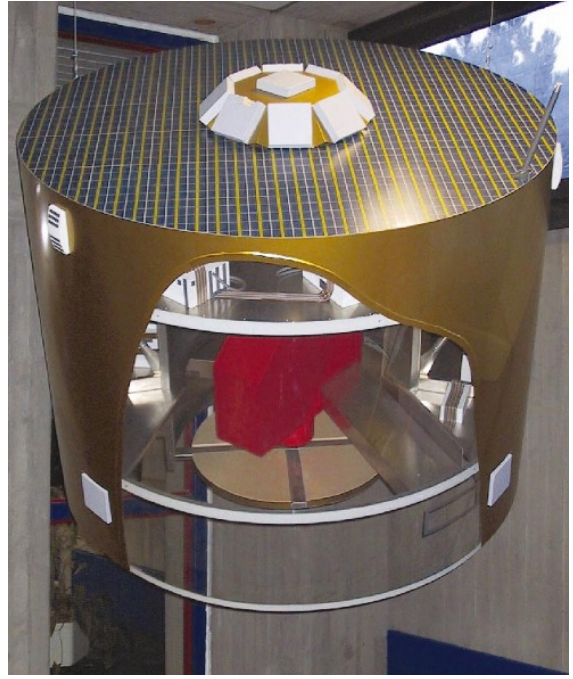

Grounded: a funding shortfall means that DIVA cannot be launched until 2007 at the earliest.

sions document the movement of stars in relation to each other, their brightness and their colour, as well as their position. Researchers can use this information to study cosmological questions - the relative motion of stars, for example, provides pointers to the age and mass of the Universe.

DIVA's main sponsor is the DLR, Germany's space agency, which had committed to paying half of the project's total cost of 57 million euros (US\$51.5 million). The rest of the money was to come from five German states, one of which pulled out, leaving a funding gap of 15 million euros. "We saw no way to close the gap," says Sigmar Wittig, the DLR's director. "So we had no choice but to slow down the mission's development and plan a launch for 2007 or 2008 ."

GAIA, meanwhile, has substantially reduced its own projected costs by planning to use a Russian rather than a European launcher, which project scientists hope will help persuade ESA to fix a launch for 2010. ESA is to decide on a new scheduling programme for all its missions early this summer.

But an early launch for GAIA would render a late launch for DIVA redundant. The same redundancy might also scotch current efforts by US scientists to revive NASA's interest in FAME.

And failure to launch either of the smaller missions before the middle of this decade would damage the whole of astrometry, says Siegfried Röser of the Institute of Computational Astronomy in Heidelberg, and lead investigator for DIVA. Young scientists will steer clear of an area of research in which no new data are expected for years on end, he says.

This prospect has led some researchers to look at the possibility of combining DIVA and FAME into a single mission. "I'd look at collaboration very favourably," says Kenneth Johnston, of the US Naval Observatory in Washington DC, FAME's principal investigator. Röser says he is not averse to such a solution, but wants to see first if ESA can be persuaded to come to DIVA's aid.

\section{No end in sight for German misconduct probe}

Marion Kerstholt, Munich

An investigation into alleged scientific misconduct at a German university has fallen far behind schedule, prompting fears that the country lacks the mechanisms needed to handle such cases.

Officials at the University of Göttingen promised 10 months ago that the investigation would be completed by September 2001. But they now admit that they are no closer to releasing its findings.

The investigation concerns a clinical trial in which patients with kidney cancer were injected with a vaccine made up of tumour cells fused with cells from the immune system.

The researchers conducting the trial claimed that the treatment caused dramatic regression of secondary tumours (A. Kugler et al. Nature Med. 6, 332-336; 2000). But the trial was suspended last year after accusations of deception and careless clinical practice were levelled in the German media at two members of the team: Alexander
Kugler, then at the University of Göttingen, and Gernot Stuhler at the University of Tübingen (see Nature 412, 8; 2001). These claims are disputed by the researchers.

Horst Kern, president of the University of Göttingen, says that the investigation into the trial has been delayed primarily by data-protection rules covering patient information. Without access to the complete files of all 18 patients involved in the trial, the external task force looking into the allegations has been unable to proceed, he says.

"We find the situation unsatisfactory," Kern says, "but we do what we can to push things along." He declines to say when the investigation will be completed.

Clinical research in Germany has come under close scrutiny since 1997 , when two biomedical researchers were accused of fabricating data in scores of papers.

Eberhard Hildt, a former postdoc at the University of Ulm, who first raised the alarm over the 1997 scandal, says it is in the interests of the scientific community for the
Before vaccination

After vaccination
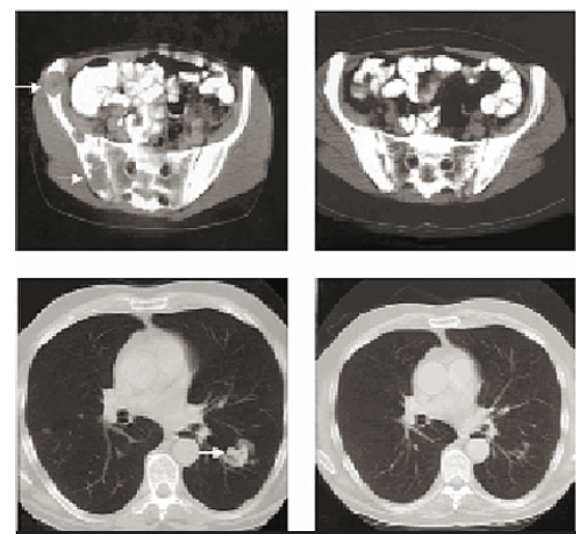

Alleged irregularities halted a vaccine trial that had reported tumour regression.

latest investigation to be completed quickly. "Data-protection problems alone are no excuse," he says. "There seem to be no similar problems with patients' data when it comes to publishing a paper.” 